\title{
Development of barley and yeast $\beta$-glucan aerogels for drug delivery by supercritical fluids
}

\author{
Marta Salgado $^{\mathrm{a}}$, Filipa Santos ${ }^{\mathrm{b}, \mathrm{c}}$, Soraya Rodríguez-Rojo ${ }^{\mathrm{a}, *}$, Rui L. Reis ${ }^{\mathrm{b}, \mathrm{c}}$, Ana Rita C. Duarte ${ }^{\mathrm{b}, \mathrm{c}}$, \\ María José Cocero ${ }^{\mathrm{a}}$ \\ ${ }^{\text {a }}$ High Pressure Processes Group, Department of Chemical Engineering and Environmental Technology, EII Sede Mergelina, University of Valladolid, 47011 Valladolid, \\ Spain \\ b 3B's Research Group- Biomaterials, Biodegradables and Biomimetics, University of Minho, Headquarters of the European Institute of Excellence on Tissue Engineering \\ and Regenerative Medicine, AvePark, 4805-017 Barco, Guimarães, Portugal \\ ' ICVS/3B's - PT Government Associate Laboratory, Braga, Guimarães, Portugal
}

\section{A R T I C L E I N F O}

\section{Keywords:}

ß-glucan

Acetylsalicylic acid

Aerogel

Rheology

Supercritical drying

Supercritical impregnation

\begin{abstract}
A B S T R A C T
Polysaccharide aerogels are a good alternative as carriers for drug delivery, since they allow high loading of the active compounds in matrices that are non-toxic, biocompatible and from a renewable feedstock. In this work, barley and yeast $\beta$-glucans aerogels were produced by gelation in aqueous solution, followed by solvent exchange and drying with supercritical $\mathrm{CO}_{2}$. First, viscoelastic properties and melting profile of the hydrogels were determined. Then, the obtained aerogels were analyzed regarding morphology, mechanical properties and behavior in physiological fluid. Both in the hydrogels and in the aerogels, big differences were observed between barley and yeast $\beta$-glucans due to their different chain structure and gelation behavior. Finally, impregnation of acetylsalicylic acid was performed at the same time as the drying of the alcogels with supercritical $\mathrm{CO}_{2}$. The release profile of the drug in PBS was analyzed in order to determine the mechanism governing the release from the $\beta$-glucan matrix.
\end{abstract}

\section{Introduction}

Aerogels are solid materials possessing low density, high porosity and high surface area. These properties allow their use in a wide variety of applications, from hydrogen storage [1] to tissue engineering [2]. Among them, aerogels are of particular interest in drug delivery of active compounds, since they offer higher loading capacity due to their surface properties $[3,4]$.

Aerogels are formed from an initial gel on aqueous phase which undergoes a drying process. Traditional drying methods, such as airdrying or freeze-drying, produce unwanted changes in the structure of the gel, leading to great shrinkage or even destruction of the network. On the contrary, drying with supercritical fluids avoids network collapse due to the absence of liquid-gas interfaces, so the porous structure is better preserved [5]. Besides, with supercritical fluids, incorporation of active compounds into the aerogel can be done simultaneously to the drying process, thus reducing processing steps and avoiding the use of organic solvents and high temperatures associated to the preparation of drug-loaded delivery systems [6]. The performance of the impregnation in supercritical fluids allows good solubility of the active compounds and diffusion through the matrix, and at the same time the structure of the matrix is well preserved. After the impregnation and upon depressurization, the final product is recovered free of any solvent and no further purification steps are required. Furthermore, supercritical impregnation enhances the penetration of the active compound into the polymeric matrix, providing a homogeneous distribution of the drug in the material [7].

To fulfill the requirements of low toxicity, biodegradability and stability for drug delivery applications, polysaccharides are a good option as carriers [8]. Many works report the production of aerogels using starch, alginate or chitin [8-14]. However, $\beta$-glucans have been barely studied for this purpose. To the author's knowledge, only Comin and coworkers produced aerogels exclusively with barley $\beta$-glucans [15]. They observed that supercritical-dried $\beta$-glucan aerogels had lower density and more homogeneous structure than the ones air-dried and freeze-dried. They also analyzed the supercritical impregnation of flax oil in the aerogels [16].

$\beta$-glucans are polymers formed by D-glucose monomers linked by $\beta$ glycosidic bonds. They can be found in cereals, algae, yeast or bacteria, with very different structures and characteristics. $\beta$-glucans have some

\footnotetext{
* Corresponding author

E-mail address: sorayarr@iq.uva.es (S. Rodríguez-Rojo).
} 
valuable features regarding processing, i.e. they increase the viscosity of solutions and can easily form hydrogels by heating $[17,18]$. Several works studied the ability of $\beta$-glucans to form gels by hydrogen bonding in junction points $[19,20]$. However, great differences are observed depending on the origin of the $\beta$-glucans, their structure, molecular weight or concentration. For instance, hydrogels are more easily created with low molecular weight $\beta$-glucans (from $20 \mathrm{kDa}$ ) because they enhance entanglement of polymer chains, whereas high molecular weight $\beta$-glucans (above $200 \mathrm{kDa}$ ) have less mobility and thus worse gelling capacity $[20,21]$.

$\beta$-glucans are being used for medical and pharmaceutical applications due to their interesting biological properties, such as wound healing ability, modulation of the immune system, anti-inflammatory or anti-bacterial properties [22,23]. In this sense, the European Food Safety Authority has recognized the ability of oat and barley $\beta$-glucans to lower blood cholesterol and thus reduce the risk of heart disease [24]. Apart from the aforementioned biological properties, some works report the protective effect of different $\beta$-glucans in oral drug delivery. On one hand, they protect the stomach against the formation of ulcers derived from intake of some drugs $[25,26]$. Further, cereal $\beta$-glucans enhance the growth of probiotics in the digestive tract [27]. On the other hand, $\beta$-glucans also protect the encapsulated active compounds through the acidic gastric medium to reach undamaged the adsorption sites in the intestine $[28,29]$. Also $\beta$-glucans from cereals and from fungi are reported to improve the permeability of active compounds through the skin into deeper layers [30,31].

In this work, $\beta$-glucan aerogels are produced by supercritical drying in $\mathrm{CO}_{2}$, aerogels were characterized and their potential as oral drug delivery systems was evaluated.

\section{Materials and methods}

\subsection{Materials}

Gels were produced from 2 types of $\beta$-glucans: barley (1-3, 1-4)- $\beta$ glucans (BBG, 75\% purity, $125 \mathrm{kDa}$ determined by size exclusion chromatography as indicated in [32], with $\beta$-glucan standards by Megazyme ranging from 40 to $359 \mathrm{kDa}$ ); Glucagel, kindly supplied by DKSH, France) and (1-3, 1-6)- $\beta$-glucans from yeast Saccharomyces cerevisae (YBG, 64\% purity, measured with $\beta$-Glucan Assay Kit (Yeast \& Mushroom), by Megazyme; L-Naturae Nutraceutical, kindly supplied by Naturae, Spain). Acetylsalicylic acid (Sigma, Portugal) was used as model active compound for the impregnation of the aerogels. PBS (pH 7.4) was prepared from tablets (Sigma). Carbon dioxide (99.998 mol\%) was supplied by Air Liquide (Portugal). All reagents were used as received.

\subsection{Production of $\beta$-glucan aerogels}

Fresh solutions of 4 and 5\% w/w BBG were produced by mixing the $\beta$-glucans with water at $80{ }^{\circ} \mathrm{C}$ for $2 \mathrm{~h}$ with stirring, until complete dissolution. Solutions with concentration lower than that were not able to create a gel. When it was completely dissolved, it was boiled for $5 \mathrm{~min}$, and then kept at $75{ }^{\circ} \mathrm{C}$ for $1 \mathrm{~h}$. The hot solution was poured into 96-well plate molds and kept overnight at $4{ }^{\circ} \mathrm{C}$ to form the gel. Longer time periods, up to $72 \mathrm{~h}$, were required as the polymer concentration decreased.

YBG was dispersed in water under stirring for $30 \mathrm{~min} .5$ and $2.5 \%$ $\mathrm{w} / \mathrm{w}$ gels were obtained after heating at $90{ }^{\circ} \mathrm{C}$ for $1 \mathrm{~h}$. After that time, the solution obtained was poured into a mold and kept at $4{ }^{\circ} \mathrm{C}$ overnight.

Gels samples were taken out from the mold and cylinders with $5 \mathrm{~mm}$ diameter and $10 \mathrm{~mm}$ height were obtained.

Hydrogels were converted into alcogels by subsequently immersing them in 20, 40, 60 and $80 \% \mathrm{v} / \mathrm{v}$ ethanol:water baths for $1.5 \mathrm{~h}$ each, and kept in pure ethanol overnight. Then they were dried with supercritical
$\mathrm{CO}_{2}$ in a critical point drier at $34^{\circ} \mathrm{C}$ and 9-9.5 $\mathrm{MPa}$, with 2 drying cycles (Autosamdri-815, Tousimis).

\subsection{Supercritical impregnation of acetylsalicylic acid}

In order to minimize processing steps, impregnation of $4 \%(\mathrm{w} / \mathrm{w})$ BBG and 2.5\% (w/w) YBG alcogels with acetylsalicylic acid was performed simultaneously to the drying of the alcogels with supercritical $\mathrm{CO}_{2}$. The alcogels were placed on a high-pressure cylinder immersed on a water bath. At the inlet, AA was placed on excess and separated from the alcogels with cotton to prevent physical contact with the alcogels. Carbon dioxide was first cooled and pumped (Haskell, MCPV-71) to the desired pressure, and then fed into the high-pressure vessel. A continuous flow of $\mathrm{CO}_{2}$ was maintained for $1.5 \mathrm{~h}$, which is the time necessary to completely remove ethanol from the structures. Due to the configuration of the drug and the polymer in the vessel, AA was first dissolved on sc- $\mathrm{CO}_{2}$. When the saturated flow of $\mathrm{CO}_{2}$ contacted the BBG and YBG matrices, they were impregnated with AA. Impregnation yield was determined at different conditions of pressure $(8,12,16$ and $20 \mathrm{MPa})$ and temperature $\left(35,40\right.$ and $\left.50{ }^{\circ} \mathrm{C}\right)$. Further information about the equipment can be found in the literature $[33,34]$.

\subsection{Rheological tests}

Viscoelastic properties of $\beta$-glucan hydrogels were evaluated on a Kinexus Prot Rheometer (Kinexus Prot, MAL1097376, Malvern) fitted with a parallel plate geometry with $10 \mathrm{~mm}$ of diameter (PU8 SR2020 SS). Oscillatory measurements were performed at $1 \%$ strain in a range on frequency of $0.01-100 \mathrm{~Hz}$ at $25^{\circ} \mathrm{C}$ in order to obtain the elastic (G') and loss (G") moduli and the complex viscosity. The thermal stability and the melting behavior of the gels was analyzed in hydrogels cured for $24 \mathrm{~h}$, through a temperature ramp with controlled frequency and strain, at $1 \mathrm{~Hz}, 1 \%$ strain and heating rate of $2.5^{\circ} \mathrm{C} / \mathrm{min}$. All measurements were performed in triplicate.

\subsection{Morphological analysis}

The produced aerogels were observed by scanning electron microscopy (SEM) with a high-resolution field emission scanning electron microscope with focus ion beam (Auriga Compact, Zeiss). The aerogels were cut in liquid nitrogen and the sections were placed by mutual conductive adhesive tape on aluminum holders and covered with gold palladium using a sputter coater.

Nitrogen adsorption-desorption isotherms were performed with ASAP 2020 (Micromeritics) to obtain surface area and pore size and volume of the aerogels. Prior to analysis, the samples were degassed at $115^{\circ} \mathrm{C}$ for $4 \mathrm{~h}$.

Aerogel density was determined with a helium pycnometer (Micromeritics Accupyc II 1340) at $25^{\circ} \mathrm{C}$ from 10 replicates (standard deviation lower than $0.5 \%$ ).

\subsection{Mechanical analysis}

Behavior of the aerogels under compression stress was analyzed with a universal testing machine (Instron 5540). Compression of the material was carried out at $1 \mathrm{~mm} / \mathrm{min}$ until the height of the sample was reduced by $70 \%$. The compressive Young modulus was determined as the initial slope in the stress-strain graphs. Aerogels were tested also after rehydrating them in PBS for $2 \mathrm{~h}$, to mimic a physiological environment. In this case, it was also possible to obtain the maximum stress that can be applied until break of the material. The tests were performed in triplicate and the results are presented as the average \pm standard deviation. 

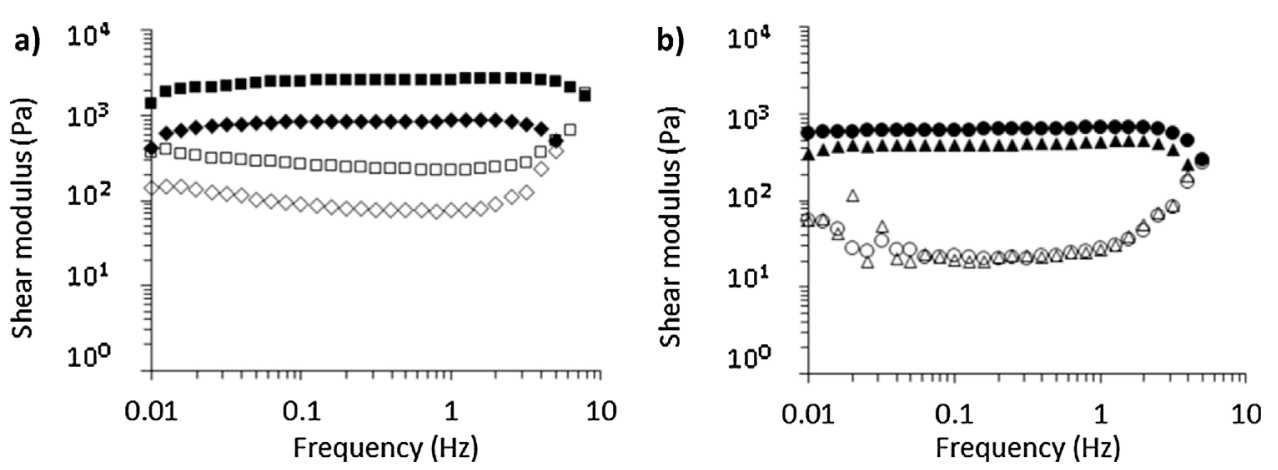

Fig. 1. G' (closed symbols) and G" (open symbols) of

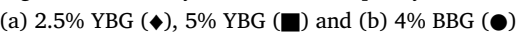
and $5 \%$ BBG ( $\mathbf{\Delta})$ hydrogels over a range of frequency in oscillatory measurements.

\subsection{Water uptake and degradation test}

Aerogel samples were placed in $5 \mathrm{~mL}$ PBS, and immersed in a stirred water bath at $37^{\circ} \mathrm{C}$. At different time points (1, 7, 14 and 21 days), samples were taken out (excess water was removed with paper) and weighted.

Water uptake was determined using the following equation:

$\%$ water uptake $=\frac{w_{w}-w_{i}}{w_{i}} \times 100$

where $w_{w}$ is the weight of the wet sample and $w_{i}$ is the weight of the initial sample.

Afterwards, the wet aerogels were changed to ethanol and dried with supercritical $\mathrm{CO}_{2}$, as indicated in Section 2.2, to ensure the complete drying of the matrix. Once the samples were dried, they were weighted $\left(\mathrm{w}_{\mathrm{f}}\right)$ to determine the weight loss, which was calculated according to the equation:

$\%$ weight loss $=\left|\frac{w_{f}-w_{i}}{w_{i}}\right| \times 100$

Water uptake and degradation test was performed in triplicate, and up to 21 days.

\subsection{Impregnation yield}

The amount of AA impregnated in the aerogels was quantified by $\mathrm{UV}$-vis at $290 \mathrm{~nm}$ using a microplate reader (Synergy HT, Bio-Tek Instruments, USA) in a quartz microplate with 96 wells (Hellma). First, the aerogels were completely dissolved in $5 \mathrm{~mL}$ PBS to ensure that all the AA was extracted from them. Then, a sample of the liquid was analyzed by UV-vis, and the absorbance was adjusted into a calibration curve between 0 and $1 \mathrm{~g} / \mathrm{L}$. The influence of BBG and YBG on the measured absorption was taken into account in the calculations by measuring the absorption obtained with an aerogel without AA and subtracting this value to the results of the absorbance of the samples with AA.

\subsection{In vitro release study}

The impregnated aerogels were placed on $5 \mathrm{~mL}$ PBS in a bath at $37^{\circ} \mathrm{C}$. Samples $(150 \mu \mathrm{L})$ of the liquid medium were taken out at different time points $(5,10,15$ and $30 \mathrm{~min}$, and $1-8$ and $24 \mathrm{~h}$ ), and replaced by the same quantity of fresh PBS. The amount of AA on the PBS at each time was measured by UV-vis spectrophotometry as mentioned before. The replacement of the aliquot with fresh PBS was taken into account in the calculations of the cumulative release of AA. All measurements were performed in triplicate.

The kinetics of release of AA was analyzed with the Power Law equation (Eq. (3)) [35]:

$\mathrm{M}_{\mathrm{t}} / \mathrm{M}_{\infty}=\mathrm{kt}^{\mathrm{n}}$
Where $M_{t}$ is the cumulative quantity of AA released at time $t, M_{\infty}$ is the theoretical amount released at infinite time (maximum AA in the aerogel), $\mathrm{k}$ is a constant characteristic of the drug-polymer system and $\mathrm{n}$ is the diffusional exponent characteristic of the release mechanism.

\section{Results and discussion}

\subsection{Rheological study of $\beta$-glucan hydrogels}

The variability on origin and chain structure between BBG and YBG leads to different behavior of both $\beta$-glucans. For instance, as it was mentioned before, BBG are soluble in water, but YBG are not. This has consequences on the gelling mechanism (upon cooling for BBG, upon heating for YBG) and chain organization of the polymer in the gels, and reflects on the production of hydrogels with different properties depending on the type of $\beta$-glucan used. The differences in the structure of the hydrogels will ultimately have influence also on the structure and properties of the final aerogels.

Fig. 1 shows the response of the different $\beta$-glucan hydrogels over a frequency range. It can be observed that for all of them the elastic gel network is maintained in a wide range of low frequencies, characterized by higher G' than G" (solid-like behavior), and both with values independent of the frequency. However, at higher frequencies, G' and G" become equal, revealing the rupture of the gel structure.

Although both $\beta$-glucans create the gel structure through junction zones due to hydrogen bonding [36,37], these are much stronger in YBG [38], and thus they resist oscillatory stress up to higher frequency (i.e. up to $4 \mathrm{~Hz}$ for $5 \% \mathrm{BBG}$, and up to $8 \mathrm{~Hz}$ for YBG). Up to the frequency values of $G^{\prime}=G^{\prime \prime}$, the gels were able to rearrange their chains around the junction points and resist shear stress, but when the frequency was further increased the junction points were damaged and the gel was broken. This behavior was also confirmed with the results of complex viscosity (Fig. 2a). Complex viscosity decreases with frequency for both $\beta$-glucans as a consequence of the rearrangement of the gels up to the values of frequency aforementioned where the structure of the gel was destroyed. Nevertheless, at low frequency, complex viscosity is higher for YBG than for $\mathrm{BBG}$, revealing more interaction between polymer chains in the YBG hydrogel.

$G$ ' increased with the concentration of YBG, indicating more elastic and more stable gel network at higher $\beta$-glucan concentration, probably due to more junction points with more quantity of polymer. This difference with concentration was not so noticeable for BBG because the range of $\beta$-glucan concentration tested was smaller. Gels of $4 \%$ BBG had almost the same moduli as $5 \%$ BBG, with the values of G' slightly higher. At 5\% $\beta$-glucan concentration, BBG had much smaller $G$ ' than YBG, indicating less elasticity of the gel. Some authors reported that molecular weight of the $\beta$-glucan has a great influence on their viscoelastic properties [21]. It is also possible that BBG polymer chains are more rigid than those of YBG, because the (1-3, 1-6)- $\beta$-glucan structure of the latter induces more voiding space between the chains, which allows better movement and rearrangement of the chains and thus more elasticity of the gel. 
a)

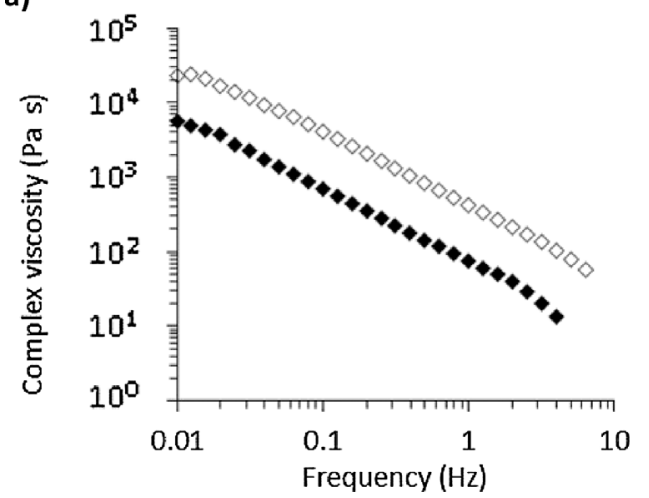

b)

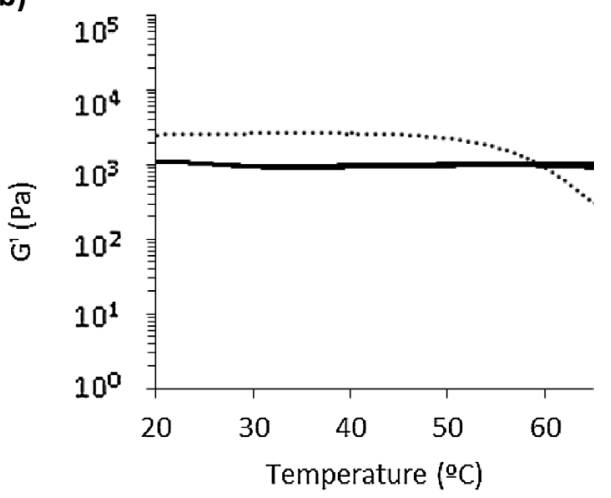

Fig. 2. (a) Complex viscosity for $5 \%(w / w)$ BBG (closed symbols) and YBG (open symbols). (b) Melting profile of 2.5\% YBG (line) and 5\% BBG (dots) hydrogels at $0.1 \%$ strain, $1 \mathrm{~Hz}$ and heating rate $3{ }^{\circ} \mathrm{C} / \mathrm{min}$.
The stability of the hydrogels was tested following the mechanical properties as a function of temperature, in a range from 20 to $70{ }^{\circ} \mathrm{C}$. Higher temperatures were not tested as water started to evaporate at this point. By heating the hydrogels (Fig. 2b) it was observed that G' started to decrease at around $50-55{ }^{\circ} \mathrm{C}$ for BBG, reflecting a loosening in the chain entanglements. This point marks the beginning of the melting of the gel. However, G' was kept constant for YBG over the range of temperature tested.

According to this result, the formation of BBG gel is a temperaturereversible process, while the gelling is irreversible for YBG upon heating. This is in accordance with the stronger hydrogen bonds in YBG hydrogel aforementioned.

\subsection{Morphological characterization of $\beta$-glucan aerogels}

$\beta$-glucan aerogels were successfully dried with supercritical $\mathrm{CO}_{2}$, preserving the structure without shrinkage during the drying process. However, the gels underwent a noticeable shrinkage during solvent exchange, especially YBG gels, because they were formed from an aqueous suspension instead of a solution (Fig. 1S). Whereas BBG formed a packed structure of polymer layers, YBG had more free space between the junction points. Thus, when solvent was changed from water to ethanol, the organization of the network was better maintained with BBG than with YBG.

All of the formed aerogels had a compact network, although the ones with lower concentration presented a more porous structure (Fig. 3). Nevertheless, the matrix was thicker with YBG, while BBG aerogels had more spongy-like structure. The same effect of the concentration and type of $\beta$-glucan was also observed by the analysis of density (Table 1). When the concentration of polymer increased, the density of the aerogels also increased, especially in the case of YBG, which had a broader concentration range. Similarly, at 5\% $\beta$-glucan, density was higher for YBG than for BBG, in accordance with the observation of thicker structure by SEM. These results were further confirmed by the mechanical tests. This might be a consequence of the different behavior of both $\beta$-glucans during hydrogel formation and solvent exchange from water to ethanol.

Surface area, pore volume and pore diameter were higher for BBG than for YBG aerogels, although with slight differences between them at the same concentration (Table 1). For each type of material, differences with the concentration could only be noticed for YBG, since the range tested was bigger. The values obtained in this work are in the range of the ones obtained with BBG by [15], except pore size, which was $2.7 \mathrm{~nm}$. The cause of this difference can be associated to the higher pressure of $\mathrm{CO}_{2}$ that they used to dry the gels, which could reduce the size of the pores and create a more uniform distribution, similarly to the changes in pore size with pressure observed in polymer foaming [39]. Also, depressurization rate is one of the parameters that influences pore size on supercritical drying of aerogels [40].

As an example, Fig. $2 \mathrm{~S}$ shows the adsorption-desorption isotherm and pore size distribution of the sample of 5\% YBG. The shape of the adsorption-desorption isotherms corresponds to a type IV isotherm, according to the IUPAC classification. This type of isotherm is characterized by a hysteresis loop, which is produced due to condensation in the capillaries. The initial part of the isotherm indicates that first there is monolayer adsorption, and, after a plateau, multilayer is formed [41]. Type IV isotherm is typical of mesoporous materials [42], and has also been observed in some other works with polysaccharide aerogels $[15,43]$. All samples had unimodal pore size distribution, although the peak was centered in $8-10 \mathrm{~nm}$ in the case of YBG and $18-22 \mathrm{~nm}$ for BBG.

\subsection{Mechanical properties}

The resistance of the aerogels to compression stress has shown to be highly dependent not only on the concentration, as it would be expected, but also on the type of $\beta$-glucan. YBG had values of compressive Young modulus almost 1-fold higher than BBG (Table 2). The linear polymer chains of BBG arranged parallel one to each other, and this kind of structure is less resistant. On the contrary, the crosslinked structure of YBG chains allowed the achievement of higher Young modulus, and thus stronger material. With both $\beta$-glucans, Young modulus increased with polymer concentration. After rehydration of the aerogels on PBS, Young modulus was greatly reduced in all cases. Although all the materials produced had low stiffness, the values are in the range of those found for other polysaccharides aerogels such as alginate, lignin or starch $[2,40]$, and are higher than others reported for BBG cryogels [36].

The different behavior of the dry and wet aerogels can be noticed by observation of the stress-strain curves. On one hand, dry aerogels had a linear region which corresponds to elastic deformation, and after some point plastic deformation occurred. In these cases, yield strain was between 10 and $15 \%$. On the other hand, the wet samples had a region of elastic deformation up to higher strain (20-30\%), but afterwards they collapsed instead of suffering plastic deformation. Fig. 3S shows, as an example, the stress-strain curves of dry and wet samples of 5\% YBG. Maximum stress at failure of the wet aerogels was in the range between 5 and $13 \mathrm{kPa}$. YBG were able to bear higher load than BBG, and also the resistance increased with the concentration of polymer. This is in agreement with the rheological behavior of the hydrogels, which revealed higher resistance to shear stress with YBG rather than BBG.

\subsection{Behavior on physiological fluids}

Water uptake has a strong influence in the release of active compounds from the matrix: if it absorbs much water, drugs can diffuse more easily to the liquid medium. To the author's knowledge, there are not previous studies about water uptake capability of $\beta$-glucan aerogels, although it has been reported for freeze-dried $\beta$-glucans. For instance, 

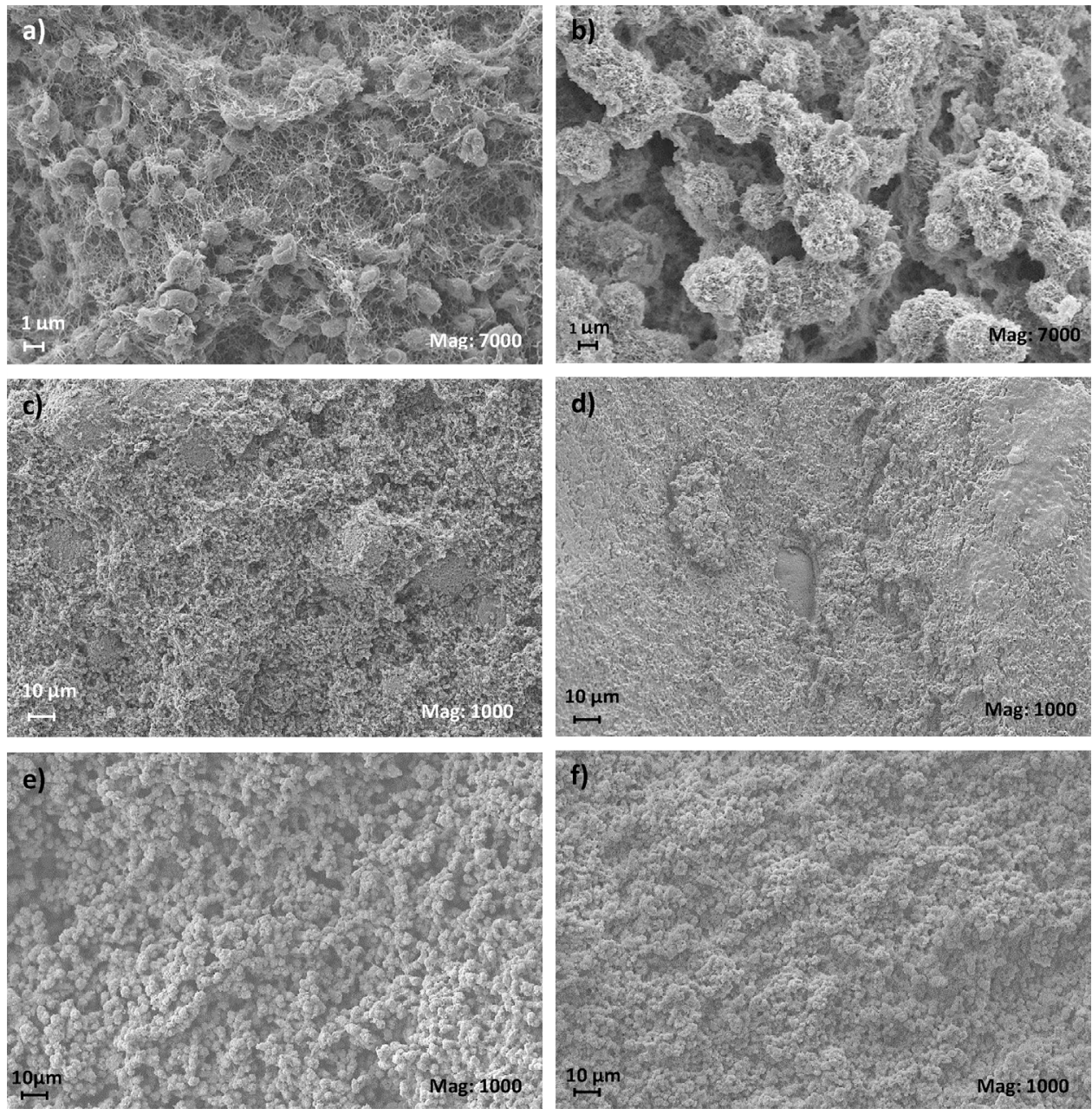

Fig. 3. SEM images of $2.5 \%$ (a and c) and $5 \%$ (d) YBG aerogels, and 4\% (b and e) and 5\% (f) BBG aerogels.
Table 1

Structural properties of the different $\beta$-glucan aerogels.

\begin{tabular}{lllll}
\hline Sample & BET surface area & Pore volume & Pore size & Density \\
\cline { 2 - 4 } & $\left(\mathrm{m}^{2} / \mathrm{g}\right)$ & $\left(\mathrm{cm}^{3} / \mathrm{g}\right)$ & $(\mathrm{nm})$ & $\left(\mathrm{kg} / \mathrm{m}^{3}\right)$ \\
& 173.1 & 0.563 & 13.7 & 34.8 \\
$2.5 \%$ YBG & 178.2 & 0.659 & 15.5 & 121.1 \\
$4 \%$ YBG & 189.4 & 0.713 & 15.8 & 69.0 \\
$5 \% B B G$ & 184.1 & 0.705 & 16.1 & 79.3 \\
\hline
\end{tabular}

Table 2

Compressive Young modulus of dry and wet $\beta$-glucan aerogels.

\begin{tabular}{lll}
\hline & \multicolumn{2}{l}{ Young modulus $(\mathrm{kPa})$} \\
\cline { 2 - 3 } & Dry & Wet \\
\hline $2.5 \%$ YBG & $286 \pm 51$ & $0.38 \pm 0.07$ \\
$5 \%$ YBG & $448 \pm 107$ & $0.36 \pm 0.08$ \\
$4 \% B B G$ & $58 \pm 14$ & $0.21 \pm 0.03$ \\
$5 \% B B G$ & $69 \pm 21$ & $0.27 \pm 0.08$ \\
\hline
\end{tabular}

Lazaridou and coworkers found that swelling and equilibrium water content of BBG lyophilized cryogels decreased with higher $\beta$-glucan molecular weight because of a more compact and less porous structure [36].

Upon soaking on physiological fluid, it was easily observed that $\beta$ glucan aerogels swelled. In all samples, the maximum water uptake was reached after $24 \mathrm{~h}$ in a physiological solution and it remained around

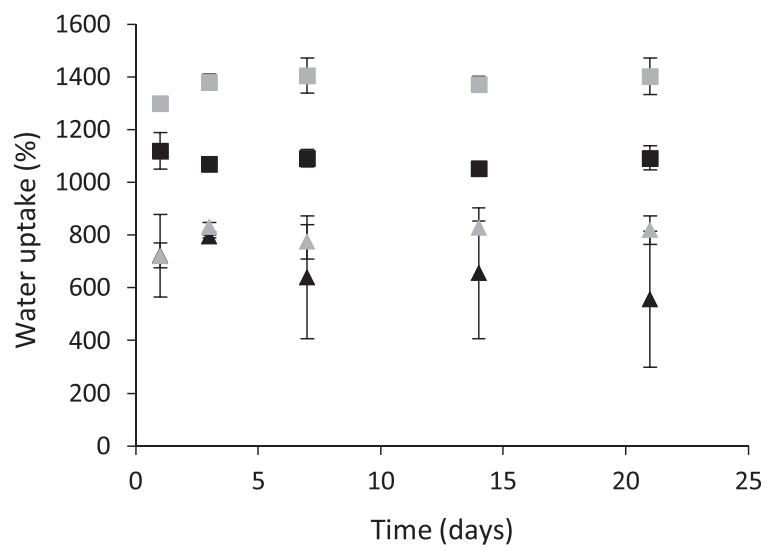

Fig. 4. Water uptake of the different $\beta$-glucan aerogel samples up to 3 weeks. Squares: YBG. Triangles: BBG. Grey: low $\beta$-glucan concentration. Black: high $\beta$-glucan concentration.

that value without significant differences for the rest of the days (Fig. 4). Besides, weight loss was lower than $20 \%$ in all cases after 21 days. This high water uptake capacity is related to the hydrophilicity of the $\beta$-glucans. Also, some previous works also reported a fast and high water uptake by other polysaccharide aerogels [2].

It was observed that, for each $\beta$-glucan, water uptake was higher with lower concentration of polymer. This is in agreement with some previous works reporting slower and smaller swelling of polysaccharide aerogels with higher polymer concentration due to the presence of more chain entanglements $[44,45]$. For $5 \% \mathrm{w} / \mathrm{w} \beta$-glucan, water uptake was 
a)

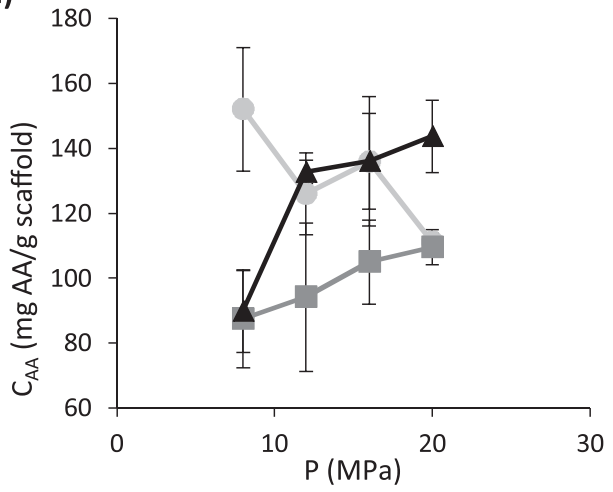

much higher for YBG.

\subsection{Supercritical impregnation of acetylsalicylic acid}

Supercritical impregnation of active compounds is composed by 2 main steps. The first one is the dissolution of the active compound in sc$\mathrm{CO}_{2}$, which depends on its solubility in $\mathrm{CO}_{2}$ (and, ultimately, on the density of $\mathrm{CO}_{2}$ ). The second step is the penetration of the active compound in the polymeric matrix, and it is influenced by the diffusion of $\mathrm{CO}_{2}$ into the structure. Both are highly dependent on the properties of supercritical $\mathrm{CO}_{2}$, and therefore pressure and temperature are key parameters in this process.

The conditions were chosen such that, temperature and pressure employed in the impregnation did not induce any changes on the morphological and mechanical parameters of the aerogels produced. The amount of AA impregnated in the aerogels depends greatly on the operating conditions and on the type of $\beta$-glucan tested (Fig. 5). In the case of BBG, at low pressure (below $12 \mathrm{MPa}$ ), impregnation yield is higher at lower temperature. The same trend with pressure and temperature is reported in the literature for the solubility of AA on sc- $\mathrm{CO}_{2}$, which presents a crossover point at around $12.5 \mathrm{MPa}$ [46]. In the case of BBG impregnated with AA, between 12 and $16 \mathrm{MPa}$, there is a crossover so that, at higher pressure, impregnation yield increases with temperature. Thus, supercritical impregnation of AA in BBG aerogels is mainly influenced by the dissolution of AA in supercritical $\mathrm{CO}_{2}$.

In the case of YBG, the impregnation yield for the same conditions of BBG shows in general terms the same order of magnitude of drug impregnated in the aerogels. However, the concentration of AA seems to decrease with the increase of the pressure, after the crossover (12.5 MPa), while in BBG we observed an increase of the concentration of aerogel with pressure. At $12 \mathrm{MPa}$, in YBG, it is observed a maximum of drug impregnation in the aerogel. After the cross-over the impregnation yield follows the expected trend, meaning a higher impregnation at higher temperatures.

When these results are related to $\mathrm{CO}_{2}$ properties (Table $1 \mathrm{~S}$ ), it is
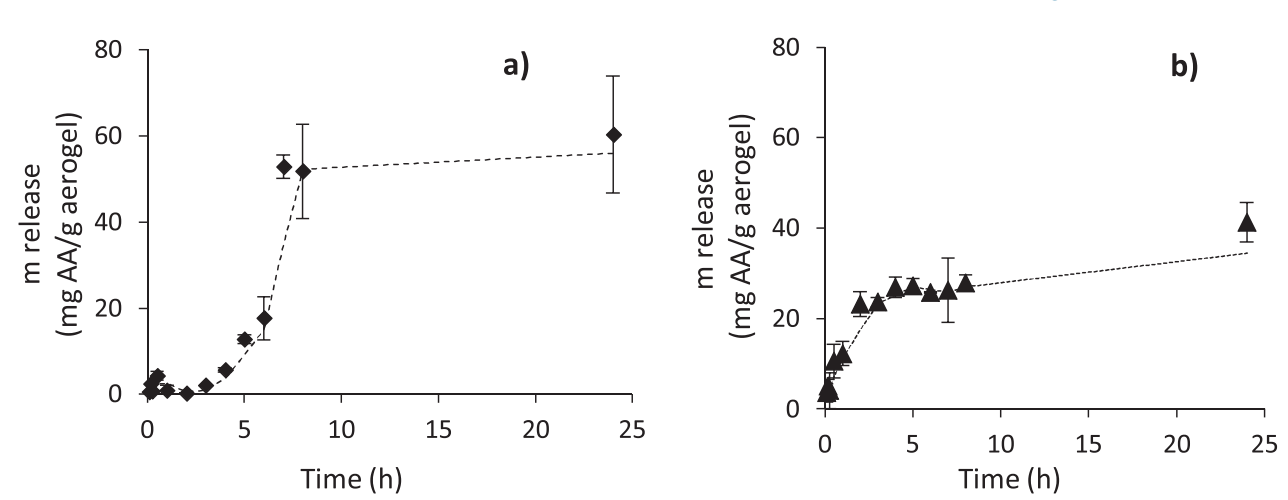

Fig. 5. Quantity of AA impregnated per mass of aerogel (4\% BBG(a) and 2.5\% YBG(b)) at different pressures and temperatures. Light grey: $35^{\circ} \mathrm{C}$; Dark grey: $40{ }^{\circ} \mathrm{C}$; Black: $50{ }^{\circ} \mathrm{C}$. observed that with BBG the quantity of AA impregnated in the aerogel has an increasing tendency with the density of $\mathrm{CO}_{2}$, because higher density enhances the solubility. Thus, in this case the impregnation is limited by the dissolution of the active compound on the $\mathrm{CO}_{2}$. On the contrary, with YBG impregnation yield decreases with density but increases with diffusivity. Hence in this case the limitation to the impregnation process is the diffusion of $\mathrm{CO}_{2}$ inside the polymeric matrix. This behavior can be also correlated with the lower surface area, pore volume and pore size of $2.5 \%$ YBG compared to $4 \%$ BBG, so that for YBG diffusion results in a key step in the impregnation process. Higher impregnation load of AA could be achieved with longer processing time, although in this work the flow of $\mathrm{CO}_{2}$ was maintained for $1.5 \mathrm{~h}$ because this was the time required for a proper drying of the alcogel.

Nevertheless, drug loading in the aerogels was in the range between 8 and $15 \%(w / w)$ in all cases. These values are higher than typical loadings of AA by supercritical impregnation reported in other previous works with different matrices (below 4\%), even though the impregnation conditions were more severe in those works $[47,48]$. However, the impregnation yield achieved in our work is comparable to that obtained for the impregnation of ketoprofen in other polysaccharides, namely alginate and starch, with similar processing conditions $[5,49]$. This is expected and it is very difficult to establish comparisons between different systems as impregnation depends on both drug and polymer and the affinity between them.

\subsection{In vitro release of acetylsalicylic acid}

The release of the active compounds from the polymeric matrix is dependent on the water uptake capacity of the polymer and the diffusion of the compound out of the matrix [50]. As both things are inherent of each material and independent of the impregnation conditions, we chose just one set of parameters to analyze the release of AA, namely $35^{\circ} \mathrm{C}$ and $8 \mathrm{MPa}$, and evaluated the release profile in simulated physiological conditions. The release profile was compared for BBG and YBG (Fig. 6).

Fig. 6. Cumulative release of acetylsalicylic acid per mass of aerogel (4\% BBG (a) and 2.5\% YBG (b)). Lines are added to guide the eye. 
The release profile of AA from BBG shows an initial period (up to $3 \mathrm{~h}$ ) of negligible release (lag time). Afterwards, from 3 to $8 \mathrm{~h}$, AA was released from the BBG matrix up to almost $60 \%$ of the total amount, and this quantity was maintained for the next $16 \mathrm{~h}$. This fast release after the lag time is related to the high water uptake capacity of the aerogel, even at short times $(24 \mathrm{~h})$.

The results obtained for release profile of AA from YBG show, on the other hand, a fast release of the drug in the first $2 \mathrm{~h}$ and then the quantity release of the drug increases slowly, presenting a more sustained release profile. The differences between the two systems may be explained by the differences encountered in the water uptake capacity of the two $\beta$-glucans. According to the results of water uptake shown previously, we observed a higher water uptake for the YBG aerogels than for the BBG aerogels, which explains the differences between the release profiles obtained (Fig. 6).

From the results obtained we confirmed that the release of AA from $2.5 \%$ YBG is more controlled than with $4 \%$ BBG. To confirm this hypothesis we have modelled the release profile curves with empirical equations, adjusted to each particular system. In the case of BBG it is necessary to take into account the lag time. The lag time depends on the thickness of the material from the surface to the active compound and not on the impregnated quantity [51]. Therefore, the initial behavior reveals a good impregnation of AA into the bulk of the $\beta$-glucan matrix, instead of being deposited just on the surface (which would be characterized by release of the active compound since the beginning). For the PBS to reach and extract AA to the liquid medium, the matrix must be first well-wetted and swelled. This delayed release can be very interesting in oral delivery, for instance when drug release is supposed to occur after a certain time since administration [52].

In order to analyze the release mechanism in the aerogels, a modification of Eq. (3) introducing the lag time (1) was required [53]:

$\mathrm{M}_{\mathrm{t}-1} / \mathrm{M}_{\infty}=\mathrm{k}(\mathrm{t}-1)^{\mathrm{n}}$

For BBG, the initial points of release during the lag time were not considered for these calculations, as well as the points corresponding to release higher than $60 \%$. By plotting $\operatorname{Ln}\left(\mathrm{M}_{\mathrm{t}-1} / \mathrm{M}_{\infty}\right)$ versus $\operatorname{Ln}(\mathrm{t}-1)$, the diffusion exponent $n$ obtained was 0.72 , with $r^{2}=0.8722$ and $k$ was $0.08 \mathrm{~h}^{-1}$.

In the case of YBG the power law (Eq. (3)) can be applied directly to the experimental data by plotting $\operatorname{Ln}\left(\mathrm{M}_{\mathrm{t}} / \mathrm{M}_{\infty}\right)$ versus $\operatorname{Ln}(\mathrm{t})$ up to $60 \%$ of the maximum drug released. The diffusional exponent obtained in this case was 0.57 with $\mathrm{r}^{2}=0.9561$, and $\mathrm{k}$ was $1.99 \mathrm{~h}^{-1}$.

For a cylindrical geometry, as it is this case, Eqs. (3) and (4) can be used in swellable cylindrical matrices $[54,55]$. However, the geometry of the matrix has to be considered in order to analyze the release mechanism governing in the system through the diffusion coefficient $n$. Thus, for cylinders, $n=0.45$ is indicative of diffusion-limiting release, $n=0.89$ define pure Case II transport (swelling-controlled release), and values in between represent anomalous release, where both diffusion and swelling influence the release. When $n$ is greater than the value of case II, the release is said to be super case II transport, with the active compound releasing freely when water penetrates the matrix. According to the modelling of the experimental data obtained from the release experiments, we can observe that the release of AA from both BBG and YBG falls in anomalous transport, which is governed both by diffusion and swelling of the matrix. This is in accordance with the behavior observed with the release profile: the release started after some initial time in which the aerogel was wetted and relaxation of the chains took place. Once the material was swollen, AA was fast released, without limitations due to diffusion of the active compound through the polymer.

\section{Conclusions}

Barley and yeast $\beta$-glucan aerogels were prepared by supercritical drying of hydrogels, after solvent exchange. Both $\beta$-glucans formed different structures due to the differences in their chain configurations: whereas the linear chains of BBG created a more rigid material, YBG arranged in a highly crosslinked configuration, which allowed easier rearrangement of the chains in the gel network. This difference in the gelation process led to re-dissolution of BBG hydrogels in water by melting, while YBG hydrogels did not present this melting effect. Besides, YBG hydrogels had more stability and elasticity than BBG ones. This also reflected in the characteristics of the aerogels. Although the morphological and structural properties of the aerogels were similar with both $\beta$-glucans, YBG had bigger density, were stronger against compression stress, and were able to absorb more water. Supercritical impregnation of acetylsalicylic acid in BBG and YBG aerogels revealed the influence of the process operating conditions on impregnation yield, which was governed by dissolution of the active compound in the case of BBG matrix and by diffusion of the compound into the matrix in the case of YBG. In the case of BBG, the release of the drug from the matrix in PBS showed an initial lag time, in which the structure was wetted and relaxation of polymer chains occurred. This delayed release could be an interesting feature for oral drug delivery in cases where a controlled release after a certain time from administration is required. For YBG, lag time was not observed, although the release achieved was more sustained. However, a deeper analysis of the dissolution of AA in acidic medium would be required.

\section{Acknowledgements}

Authors acknowledge Ministerio de Economía y Competitividad (MINECO) through project CTQ2013-44143-R and project PIP 063/ 147181 from Fundación General of the University of Valladolid for financial support. M. Salgado thanks to Ministerio de Educación, Ciencia y Deporte (MECD) for her FPU and mobility grants. S. Rodríguez-Rojo acknowledges to MINECO and UVa for her Juan de la Cierva fellowship (JCI-2012-14992). The research leading to these results has received funding from the European Union Seventh Framework Programme (FP7/2007-2013) under grant agreement number REGPOT-CT2012316331-POLARIS and from the project "Novel smart and biomimetic materials for innovative regenerative medicine approaches" RL1 ABMR - NORTE-01-0124-FEDER-000016) co-financed by North Portugal Regional Operational Programme (ON.2-O Novo Norte), under the National Strategic Reference Framework (NSRF), through the European Regional Development Fund (ERDF).

\section{References}

[1] M. Rueda, L.M. Sanz-Moral, A. Nieto-Márquez, P. Longone, F. Mattea, Á. Martín, Production of silica aerogel microparticles loaded with ammonia borane by batch and semicontinuous supercritical drying techniques, J. Supercrit. Fluids 92 (2014) 299-310.

[2] S. Quraishi, M. Martins, A.A. Barros, P. Gurikov, S.P. Raman, I. Smirnova, A.R.C. Duarte, R.L. Reis, Novel non-cytotoxic alginate-lignin hybrid aerogels as scaffolds for tissue engineering, J. Supercrit. Fluids 105 (2015) 1-8.

[3] C.A. García-González, M. Alnaief, I. Smirnova, Polysaccharide-based aerogels-Promising biodegradable carriers for drug delivery systems, Carbohydr. Polym. 86 (4) (2011) 1425-1438.

[4] Z. Ulker, C. Erkey, An emerging platform for drug delivery: aerogel based systems, J. Controlled Release 177 (2014) 51-63.

[5] C.A. García-González, J.J. Uy, M. Alnaief, I. Smirnova, Preparation of tailor-made starch-based aerogel microspheres by the emulsion-gelation method, Carbohydr. Polym. 88 (4) (2012) 1378-1386.

[6] M. Champeau, J.M. Thomassin, T. Tassaing, C. Jérôme, Drug loading of polymer implants by supercritical $\mathrm{CO}_{2}$ assisted impregnation: a review, J. Controlled Release 209 (2015) 248-259.

[7] Y. Masmoudi, L. Ben Azzouk, O. Forzano, J.M. Andre, E. Badens, Supercritical impregnation of intraocular lenses, J. Supercrit. Fluids 60 (2011) 98-105.

[8] H. Maleki, L. Durães, C.A. García-González, P. del Gaudio, A. Portugal, M. Mahmoudi, Synthesis and biomedical applications of aerogels: possibilities and challenges, Adv. Colloid Interface Sci. 236 (2016) 1-27.

[9] A.N. Mustapa, A. Martin, L.M. Sanz-Moral, M. Rueda, M.J. Cocero, Impregnation of medicinal plant phytochemical compounds into silica and alginate aerogels, J. Supercrit. Fluids (2016).

[10] T. Mehling, I. Smirnova, U. Guenther, R.H.H. Neubert, Polysaccharide-based 
aerogels as drug carriers, J. Non Cryst. Solids 355 (50-51) (2009) 2472-2479.

[11] R.A.A. Muzzarelli, Biomedical exploitation of chitin and chitosan via mechanochemical disassembly, electrospinning, dissolution in imidazolium ionic liquids, and supercritical drying, Mar. Drugs 9 (9) (2011) 1510.

[12] I. De Marco, E. Reverchon, Starch aerogel loaded with poorly water-soluble vitamins through supercritical CO2 adsorption, Chem. Eng. Res. Des. 119 (2017) 221-230.

[13] L. Baldino, S. Concilio, S. Cardea, E. Reverchon, Interpenetration of natural polymer aerogels by supercritical drying, Polymers 8 (4) (2016).

[14] S. Cardea, L. Baldino, I.D. Marco, P. Pisanti, E. Reverchon, Supercritical gel drying of polymeric hydrogels for tissue engineering applications, Chem. Eng. Trans. 32 (2013) 1123-1128.

[15] L.M. Comin, F. Temelli, M.D.A. Saldaña, Barley beta-glucan aerogels via supercritical $\mathrm{CO}_{2}$ drying, Food Res. Int. 48 (2) (2012) 442-448.

[16] L.M. Comin, F. Temelli, M.D.A. Saldaña, Barley $\beta$-glucan aerogels as a carrier for flax oil via supercritical $\mathrm{CO}_{2}$, J. Food Eng. 111 (4) (2012) 625-631.

[17] Y. Liu, J. Zhang, Q. Tang, Y. Yang, Y. Xia, S. Zhou, D. Wu, Z. Zhang, L. Dong, S.W. Cui, Rheological properties of $\beta$-d-glucan from the fruiting bodies of Ganoderma lucidum, Food Hydrocolloids 58 (2016) 120-125.

[18] A. Lazaridou, C.G. Biliaderis, M.S. Izydorczyk, Molecular size effects on rheological properties of oat $\beta$-glucans in solution and gels, Food Hydrocolloids 17 (5) (2003) 693-712.

[19] A. Lazaridou, C.G. Biliaderis, Molecular aspects of cereal $\beta$-glucan functionality: physical properties, technological applications and physiological effects, J. Cereal Sci. 46 (2) (2007) 101-118.

[20] S.M. Tosh, P.J. Wood, Q. Wang, J. Weisz, Structural characteristics and rheological properties of partially hydrolyzed oat $\beta$-glucan: the effects of molecular weight and hydrolysis method, Carbohydr. Polym. 55 (4) (2004) 425-436.

[21] Y. Brummer, C. Defelice, Y. Wu, M. Kwong, P.J. Wood, S.M. Tosh, Textural and rheological properties of oat beta-glucan gels with varying molecular weight composition, J. Agric. Food Chem. 62 (14) (2014) 3160-3167.

[22] J. Chen, R. Seviour, Medicinal importance of fungal $\beta$-( $1 \rightarrow 3)$, $(1 \rightarrow 6)$-glucans, Mycol. Res. 111 (6) (2007) 635-652.

[23] B. Du, Z. Bian, B. Xu, Skin health promotion effects of natural beta-glucan derived from cereals and microorganisms: a review, Phytother. Res. 28 (2) (2014) 159-166.

[24] N. Efsa, Panel on Dietetic Products, Allergies, Scientific Opinion on the substantiation of a health claim related to oat beta glucan and lowering blood cholesterol and reduced risk of (coronary) heart disease pursuant to Article 14 of Regulation (EC) No 1924/2006, EFSA J. 8 (12) (2010).

[25] D. Suchecka, J. Harasym, J. Wilczak, J. Gromadzka-Ostrowska, Hepato- and gastroprotective activity of purified oat 1-3, 1-4- $\beta$-d-glucans of different molecular weight, Int. J. Biol. Macromol. 91 (2016) 1177-1185.

[26] O.V. Ozkan, O.H. Ozturk, M. Aydin, N. Yilmaz, I. Yetim, A. Nacar, S. Oktar, S. Sogut, Effects of $\beta$-glucan pretreatment on acetylsalicylic acid-induced gastric damage: an experimental study in rats, Curr. Ther. Res. 71 (6) (2010) 369-383.

[27] K.-L. Lam, P. Chi-Keung Cheung, Non-digestible long chain beta-glucans as novel prebiotics, Bioact. Carbohydr. Dietary Fibre 2 (1) (2013) 45-64.

[28] A. Shah, A. Gani, M. Ahmad, B.A. Ashwar, F.A. Masoodi, $\beta$-Glucan as an encapsulating agent: effect on probiotic survival in simulated gastrointestinal tract, Int. J. Biol. Macromol. 82 (2016) 217-222.

[29] R. De Smet, T. Demoor, S. Verschuere, M. Dullaers, G.R. Ostroff, G. Leclercq, L. Allais, C. Pilette, M. Dierendonck, B.G. De Geest, C.A. Cuvelier, ß-Glucan microparticles are good candidates for mucosal antigen delivery in oral vaccination, $\mathrm{J}$. Controlled Release 172 (3) (2013) 671-678.

[30] B. Klein, $\beta$-D-glucan topical composition, WO1999021531 A1, 1999.

[31] J. Röding, $\beta-(1,3)-\beta-(1,4)$-glucan as carrier for chemical substances, WO 2006015627 A1, 2006.

[32] M. Salgado, S. Rodríguez-Rojo, F.M. Alves-Santos, M.J. Cocero, Encapsulation of resveratrol on lecithin and $\beta$-glucans to enhance its action against Botrytis cinerea, J. Food Eng. 165 (2015) 13-21.

[33] A.R.C. Duarte, M.S. Costa, A.L. Simplício, M.M. Cardoso, C.M.M. Duarte,
Preparation of controlled release microspheres using supercritical fluid technology for delivery of anti-inflammatory drugs, Int. J. Pharm. 308 (1-2) (2006) 168-174.

[34] V.P. Costa, M.E.M. Braga, J.P. Guerra, A.R.C. Duarte, C.M.M. Duarte, E.O.B. Leite, M.H. Gil, H.C. de Sousa, Development of therapeutic contact lenses using a supercritical solvent impregnation method, J. Supercrit. Fluids 52 (3) (2010) 306-316.

[35] P.L. Ritger, N.A. Peppas, A simple equation for description of solute release I. Fickian and non-fickian release from non-swellable devices in the form of slabs, spheres, cylinders or discs, J. Controlled Release 5 (1) (1987) 23-36.

[36] A. Lazaridou, K. Kritikopoulou, C.G. Biliaderis, Barley $\beta$-glucan cryogels as encapsulation carriers of proteins: impact of molecular size on thermo-mechanical and release properties, Bioact. Carbohydr. Dietary Fibre 6 (2) (2015) 99-108.

[37] X. Zheng, S. Zou, H. Xu, Q. Liu, J. Song, M. Xu, X. Xu, L. Zhang, The linear structure of (-glucan from baker's yeast and its activation of macrophage-like RAW264.7 cells, Carbohydr. Polym. 148 (2016) 61-68.

[38] H. Liu, Y. Li, J. Gao, A. Shi, L. Liu, H. Hu, N. Putri, H. Yu, W. Fan, Q. Wang, Effects of microfluidization with ionic liquids on the solubilization and structure of $\beta$-dglucan, Int. J. Biol. Macromol. 84 (2016) 394-401.

[39] E. Reverchon, S. Cardea, Production of controlled polymeric foams by supercritical $\mathrm{CO}_{2}$, J. Supercrit. Fluids 40 (1) (2007) 144-152.

[40] M. Martins, A.A. Barros, S. Quraishi, P. Gurikov, S.P. Raman, I. Smirnova, A.R.C. Duarte, R.L. Reis, Preparation of macroporous alginate-based aerogels for biomedical applications, J. Supercrit. Fluids 106 (2015) 152-159.

[41] S. Lowell, J.E. Shields, Adsorption isotherms, Powder Surface Area and Porosity, Springer, Netherlands, Dordrecht, 1984 pp. 11-13.

[42] A. Romero, E. Alonso, Á. Sastre, A. Nieto-Márquez, Conversion of biomass into sorbitol: cellulose hydrolysis on MCM-48 and d-Glucose hydrogenation on Ru/ MCM-48, Microporous Mesoporous Mater. 224 (2016) 1-8.

[43] F. Quignard, R. Valentin, F. Di Renzo, Aerogel materials from marine polysaccharides, New J. Chem. 32 (8) (2008) 1300-1310.

[44] F. De Cicco, P. Russo, E. Reverchon, C.A. García-González, R.P. Aquino, P. Del Gaudio, Prilling and supercritical drying: a successful duo to produce core-shell polysaccharide aerogel beads for wound healing, Carbohydr. Polym. 147 (2016) 482-489.

[45] D. Bilanovic, J. Starosvetsky, R.H. Armon, Preparation of biodegradable xanthan-glycerol hydrogel, foam, film, aerogel and xerogel at room temperature, Carbohydr. Polym. 148 (2016) 243-250.

[46] Z. Huang, W.D. Lu, S. Kawi, Y.C. Chiew, Solubility of aspirin in supercritical carbon dioxide with and without acetone, J. Chem. Eng. Data 49 (5) (2004) 1323-1327.

[47] A.R.C. Duarte, T. Casimiro, A. Aguiar-Ricardo, A.L. Simplício, C.M.M. Duarte, Supercritical fluid polymerisation and impregnation of molecularly imprinted polymers for drug delivery, J. Supercrit. Fluids 39 (1) (2006) 102-106.

[48] C. Domingo, J. García-Carmona, M.A. Fanovich, J. Saurina, Study of adsorption processes of model drugs at supercritical conditions using partial least squares regression, Anal. Chim. Acta 452 (2) (2002) 311-319.

[49] A.A. Barros, C. Oliveira, R.L. Reis, E. Lima, A.R.C. Duarte, Ketoprofen-eluting biodegradable ureteral stents by $\mathrm{CO}_{2}$ impregnation: in vitro study, Int. J. Pharm. 495 (2) (2015) 651-659.

[50] A.R.C. Duarte, J.F. Mano, R.L. Reis, Dexamethasone-loaded scaffolds prepared by supercritical-assisted phase inversion, Acta Biomater. 5 (6) (2009) 2054-2062.

[51] C.-C. Kao, S.-C. Chen, M.-T. Sheu, Lag time method to delay drug release to various sites in the gastrointestinal tract, J. Controlled Release 44 (2-3) (1997) 263-270.

[52] A. Gazzaniga, L. Palugan, A. Foppoli, M.E. Sangalli, Oral pulsatile delivery systems based on swellable hydrophilic polymers, Eur. J. Pharm. Biopharm. 68 (1) (2008) $11-18$.

[53] P. Costa, J.M. Sousa Lobo, Modeling and comparison of dissolution profiles, Eur. J. Pharm. Sci. 13 (2) (2001) 123-133.

[54] K. Kosmidis, E. Rinaki, P. Argyrakis, P. Macheras, Analysis of Case II drug transport with radial and axial release from cylinders, Int. J. Pharm. 254 (2) (2003) 183-188.

[55] H. Park, K. Park, W.S.W. Shalaby, Biodegradable Hydrogels for Drug Delivery, Taylor \& Francis, 2011. 